\title{
Remotely monitored inactivity due to COVID-19 lockdowns
}

\author{
Potential hazard for heart failure patients
}

\author{
Ahmed Al Fagih, MD, Mariam Al Onazi, RCES, Saleh Al Basiri, MD, Fahmi AL-Kaf, MD, Khalid Dagriri, MD, \\ Yahya Al Hebaishi, MD, Sondos Samargandy, MD, Lamia Al Shengeiti, MD.
}

\begin{abstract}

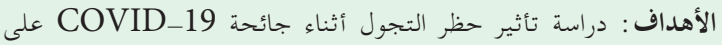

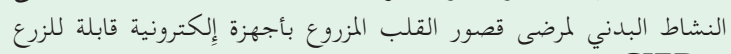
.(CIEDs)

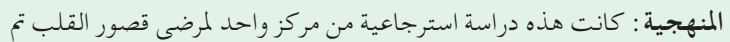

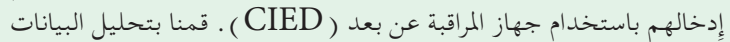

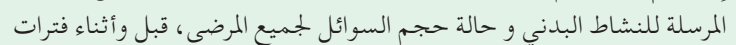

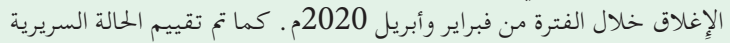
للمرضى.

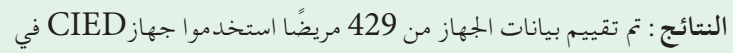

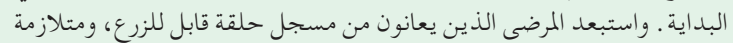

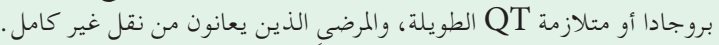

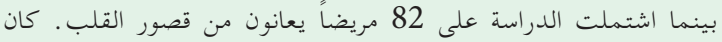

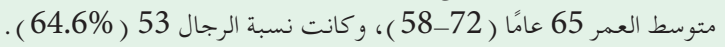

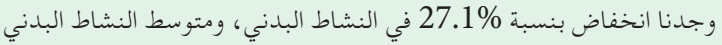

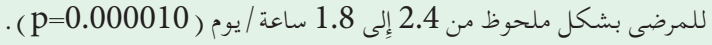

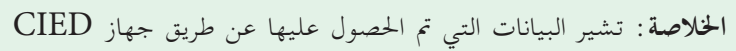

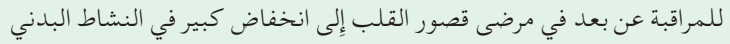

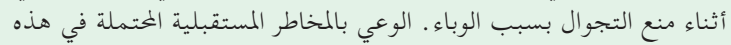

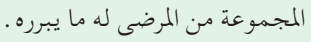

Objectives: To study the impact of curfews during the COVID-19 pandemic, on the physical activity in patients of heart failure implanted withcardiac implantable electronic devices (CIEDs).

Methods: This was a retrospective single-center study of heart failure patients inserted with remote monitoring (RM)-capable CIED. We analyzed the transmitted data of physical activity and fluid volume status of all patients, before, and during the lockdown periods between February and April 2020. The clinical status of the patients was also evaluated.
Results: Device data from 429 patients implanted with CIED capable of RM were initially evaluated. Patients with an implantable loop recorder, Brugada or Long QT syndromes, and patients with incomplete transmissions were excluded. Eighty-two patients with heart failure were included. The median age was 65 years (58-72), and $53(64.6 \%)$ subjects were men. There was a $27.1 \%$ decline in physical activity, and the median physical activity of patients significantly declined from 2.4 to 1.8 hours/day $(p=0.000010)$.

Conclusion: Data obtained by remotely monitored CIED in heart failure patients suggests a significant decline in physical activity during the country lockdown due to the pandemic. Awareness of the future potential hazards in this group of patients is warranted.

Keywords: COVID-19, CRT, ICD, physical activity, remote monitoring

Saudi Med J 2020; Vol. 41 (11): 1211-1216 doi: 10.15537/smj.2020.11.25449

From the Electrophysiology Division, Adult Cardiology Department (Al Fagih, Al Onazi, Al Basiri, AL-Kaf, Dagriri, Al Hebaishi, Al Shengeiti), and from the Interventional Cardiology Division, Adult Cardiology Department (Samargandy), Prince Sultan Cardiac Center, Riyadh, Kingdom of Saudi Arabia.

Received 29th June 2020. Accepted 21st September 2020.

Address correspondence and reprint request to: Dr. Ahmed Al Fagih, Consultant, Electrophysiology Division, Adult Cardiology Department, Prince Sultan Cardiac Center, Riyadh, Kingdom of Saudi Arabia. E-mail:aafagih@yahoo.com

ORCID ID: https://orcid.org/0000-0002-7765-0699 
$\mathrm{C}$ ardiac implantable electronic devices (CIEDs) with remote monitoring (RM) capabilities might be beneficial in the clinical management of heart failure (HF) patients in the COVID-19 era. It allows early detection of arrhythmias, pre-clinical HF indicators, and technical device follow-up. ${ }^{1}$ In addition, most of the recent generations of CIED continuously collect and store daily physical activity data through a built-in accelerometer, thus providing a new opportunity to assess the patient activity remotely. ${ }^{2}$ Current evidence suggests that device-based measured physical activity combined with other device parameters are accurate and might predict the clinical outcomes in $\mathrm{HF}$ patients. ${ }^{3}$ The World Health Organization (WHO) has established clear guidelines on the minimal amount of physical activity necessary to maintain adequate health and fitness. ${ }^{4}$ In order to provide appropriate care for patients living with CIED while limiting exposure to the hospital staff during the COVID-19 pandemic, the Heart Rhythm Society COVID-19 Task Force update document stated that every effort should be made to perform CIED interrogation via RM rather than in-person visits. ${ }^{5}$

We objectively evaluate the impact of public health measures such as curfews and lockdowns due to the COVID-19 pandemic on physical activity in $\mathrm{HF}$ patients.

Methods. This was a retrospective single-center study conducted at Prince Sultan Cardiac Center, Riyadh, Kingdom of Saudi Arabia, including $\mathrm{HF}$ patients with reduced ejection fraction $(<40 \%)$. The study was approved by our institution's Research \& Ethics Committee (R20016), and conformed to the Declaration of Helsinki. Verbal informed consent was obtained before the interview during a telemedicine appointment at our institution's outpatient clinics.

Heart failure patients having a left ejection fraction less than $40 \%$ were implanted with various models of RM-capable CIED Medtronic devices (Medtronic, Dublin, Ireland) were recruited in the study. Patients who received implantable cardioverter defibrillators or cardiac resynchronization therapy (ICD/CRT) were included. Patients who had an implantable loop recorder, Brugada and/or Long QT syndromes, technical problems in device transmissions, history of

Disclosure. Authors have no conflict of interests, and the work was not supported or funded by any drug company. impaired physical limitation, poor mobility, or received ICD/CRT device less than 6 months ago were excluded. Medtronic ICD/CRT devices contain a single-axis accelerometer that records patient activity and is graphically reported as hours per day in the cardiac compass report. ${ }^{6}$

Data including patient demographics such as age, gender, residence, comorbidities, and the implanted device type, were collected. A structured questionnaire was developed by the investigators to determine the clinical status of patients during the period of lockdown such as New York Heart Association Class, palpitation, presence of dizziness or syncope, adjusted use of diuretics, unplanned hospitalization, and the amount of daily activity and exercise. Physical activity levels were defined as hours per day (hrs/day). Thoracic impedance (Ohms), and Optivol index reflecting the fluid status of patients were collected from the Medtronic CareLink ${ }^{\circ}$ Network from February 2 to April 19, 2020. Device generated data were obtained across the following durations: pre-lockdown period from February 2, 2020 to March 12, 2020 while the lockdown period was from March 12, 2020 to April 19, 2020. Data was assessed in the center virtual device clinic, which has full access to all remotely monitored parameters. All remotely monitored variables before and throughout the lockdown periods were compared.

Statistical analysis was performed by comparing the changes in 2 different time periods: before and during implementation of the lockdown. Patient characteristics were summarized using descriptive statistics (Table 1). Categorical variables were reported as frequencies and percentages. Continuous variables were expressed as mean \pm standard deviation or median with the 25 th and 75th percentiles. Visualization of Q-Q plots and Shapiro-Wilk test was used to assess the normality distribution of continuous variables. Differences in physical activity among males and females were compared using the Mann-Whitney $U$ test. The difference in physical activity between the first and last day of the study period was assessed using the RelatedSamples Wilcoxon Rank Signed test. All statistical and graphical analyses were performed using IBM-SPSS version 25 (IBM Corp., Armonk, N.Y.). A $p$-value of 0.05 was considered statistically significant.

Results. A total of 429 patients implanted with RM capable CIED in our center were initially evaluated. Eighty-two patients were included in the analysis after careful review of the inclusion and exclusion criteria. All patients had valid data transmitted throughout the study period. The median age of patients was 65 years 
(58-72), and 53 (64.6\%) were men. Demographic data and comorbidities of the patients are summarized in Table 1.

Remotely monitored data. During the first week of the study, the median physical activity of male patients (2.5 [1.3-5.1] hrs/day) was higher than that of females (2 [1-3.9] hrs/day), although it was not statistically significant $(p=0.571)$. However, during the last week of the study, both gender showed a relatively similar decline in physical activity, (1.8 [1-3.3] hrs/day) among males and (1.5 [0.8-3] hours/day) among females.

At the end of the study period, there was a $27.1 \%$ decline in the physical activity of HF patients. Comparing the first week of February 2020 and the last week of March of the same year until the end of the study period, the median physical activity of patients declined from 2.4 to $1.8 \mathrm{hrs} /$ day $(p=0.000010)$. The change in the trend of physical activity occurred in the first week of March 2020, which coincides with the implementation of social distancing measures around the country (Figure 1). The measured thoracic impedance and Optivol index did not show a significant change in the trend throughout the study period (Figure 2).

Patients' symptoms during curfew. The clinical status of the patients was assessed using a structured questionnaire. Twenty-seven (32.9\%) patients were found to have NYHA class II and III. Eight patients $(9.8 \%)$ reported emergency room visits and one $(1.2 \%)$ patient had an unplanned hospitalization for HF symptoms. Ten (12.2\%) patients required diuretic dose adjustment. Forty-two $(51.2 \%)$ patients reported that they maintained their level of exercise throughout the study period. None of the patients reported experiencing typical symptoms nor tested positive for COVID-19.

Discussion. Since its introduction, CIED remote monitoring has provided convenience and comparative

Table 1 - Demographic data and medical history of 82 patients.

\begin{tabular}{lc}
\hline Variables & $\begin{array}{c}\mathbf{n}(\%) \text { or median } \\
\left(25^{\text {th }}, 75^{\text {th }} \text { percentile }\right)\end{array}$ \\
\hline Age, years & $65(58,72)$ \\
Male & $53(64.6)$ \\
Weight, Kg & $80.5(72,90)$ \\
Diabetes & $46(56.1)$ \\
Hypertension & $34(41.5)$ \\
Ischemic heart disease & $36(49.9)$ \\
Ejection fraction, \% & $30(23.8,41.2)$ \\
Medications & \\
ACE inhibitors & $28(34.1)$ \\
Angiotensin II receptor blockers & $43(52.4)$ \\
Beta blockers & $81(98.8)$ \\
Anti-arrhythmic & $21(25.6)$ \\
Diuretic & $71(86.6)$ \\
Warfarin & $9(11.0)$ \\
NOAC & $13(15.9$ \\
\hline ACE: angiotensin converting enzyme, NOAC: non-vitamin K oral & \\
& anticoagulants
\end{tabular}

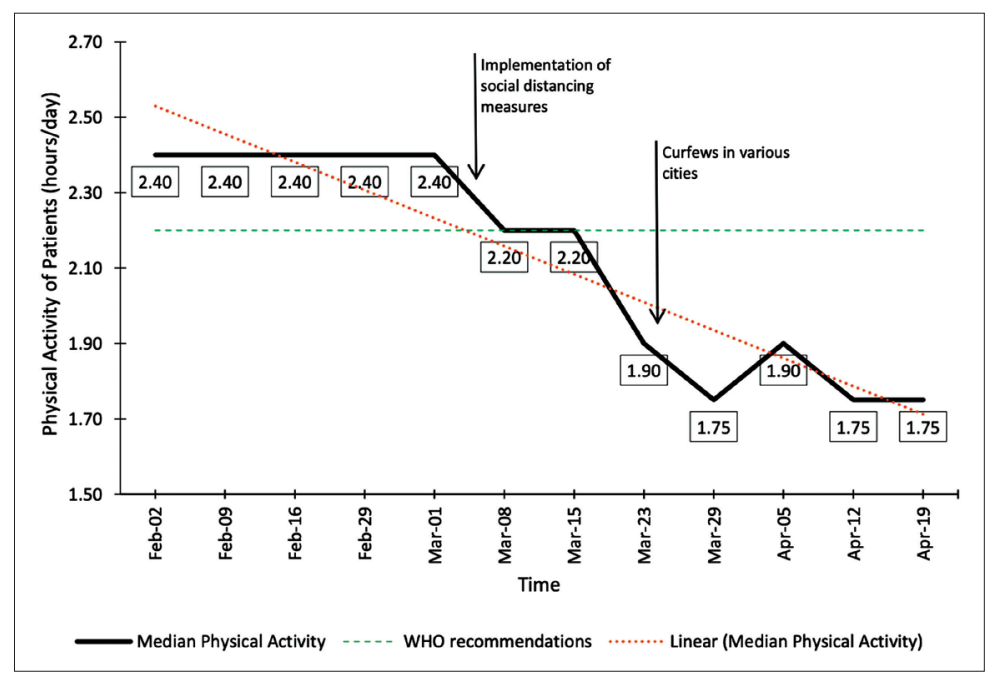

Figure 1 - Median plots of heart failure patients' average weekly activity from February to April 2020. 
advantages over in-person evaluation, especially for patients with $\mathrm{HF}^{7-9}$ It is feasible and has clear implications for the clinical follow-up of implantable devices as well as disease and medical management. ${ }^{10-12}$ Patient's physical activity as assessed by ICD/CRT devices has intra-individual variation based on the mode of exercise. ${ }^{13}$ Various forms of lockdown have been implemented by governments in an attempt to prevent the spread of the novel coronavirus disease 2019 (COVID-19) worldwide. ${ }^{14}$ It is vital to understand the impact of such public health recommendations on physical activity in HF patients as well as the expected consequences on their clinical outcome. ${ }^{15}$ Although the differences in overall physical activity might be attributed to seasonal variations, the decline in the activity of our patients coincides with the announcement of the social distancing recommendations and curfew in the country. ${ }^{16,17}$ In a very recent Canadian study, children with congenital heart disease had a significant decline in their daily physical activity assessed by step-count during the COVID-19 pandemic compared to that during the same season of the preceding year. ${ }^{18}$ Social

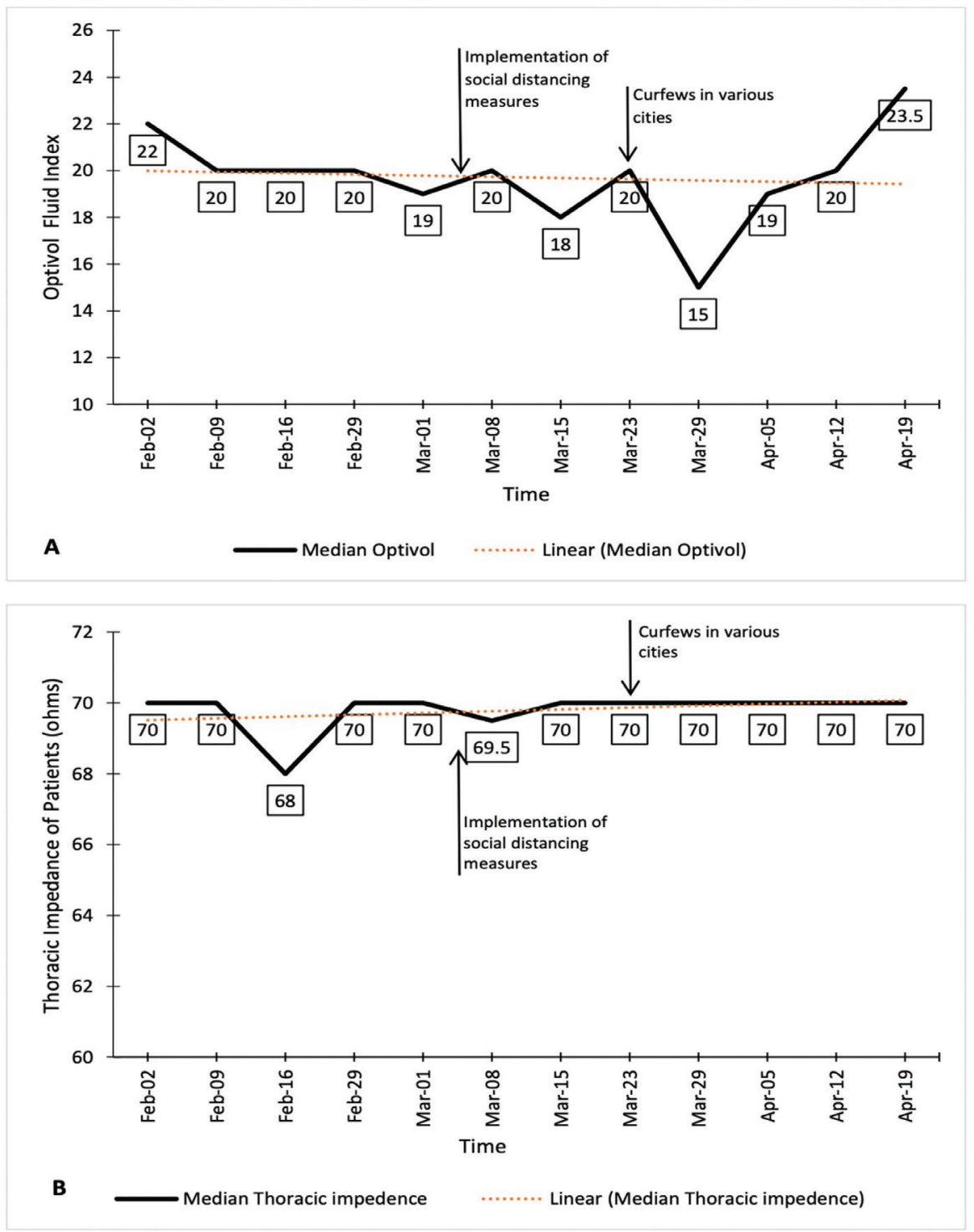

*OptiVol 2.0 fluid index is an accumulation of the difference between the daily and reference impedance

Figure 2 - Median plots of A) Optivol fluid index and B) thoracic impedance. 
distancing measures were introduced in our country gradually in early March 2020, followed by curfew in most cities within 2 weeks. ${ }^{17}$ The impact of these public health recommendations was clearly reflected in our HF patients (Figure 1). Despite the significant decline in physical activity, the measured thoracic impedance and Optivol index were maintained and did not show a significant change throughout the study period (Figure 2). This could explain why only one patient required unplanned admission for HF symptoms, and few patients required diuretic dose adjustment. Yet, the long-term effect of such a situation is not clear. A significant drop in thoracic impedance sufficient to generate a fluid index maintained above the threshold level for more than 30 days per year was associated with significantly higher rates of hospitalization for acute decompensated HF. ${ }^{19}$ Low levels of physical activity in HF patients might also predispose to the development of atrial arrhythmia and increase the morbidity and mortality. ${ }^{20,21}$ In one study of CRT patients, a decline in the device measured physical activity of more than $40 \%$ was found to be a short-term predictor of mortality, HF events, and ventricular tachyarrhythmia. ${ }^{22}$ Activity reduced by 30 minutes per day in a given month has an estimated $48 \%$ higher risk of death at 4 years compared to a similar patient in the same month. ${ }^{23}$

Study limitations. First, it is a single-center study, resulting in a limited number of patients recruited. A limited number of patients might contribute to a non-statistically significant result despite their clinical significance. Second, its retrospective design might have been affected by uncontrolled factors; therefore, a single factor affecting the physical activity of the patients might affect the data at any point during the study period.

In conclusion, in the light of a significant decline in physical activity during COVID-19 as evidenced by remotely monitored CIED, the potential long-term impacts of public health precautions in response to the pandemic, especially among patients with HF needs to be considered.

Acknowledgment. We would like to thank Editage (www. editage.com) for English language editing.

\section{References}

1. Kawabata M, Fantoni C, Regoli F, Raffa S, Pastori F, Fratini S, et al. Activity monitoring in heart failure patients with cardiac resynchronization therapy. Circ J 2007; 71: 1885-1892.

2. Kaszala K, Ellenbogen KA. Device Sensing. Circulation 2010; 122: $1328-1340$.
3. Rosman L, Lampert R, Sears SF, Burg MM. Measuring Physical Activity With Implanted Cardiac Devices: A Systematic Review. Journal of the American Heart Association 2018; 7: e008663.

4. World Health Organization. Physical activity [Update 2018 February 23; Accessed 2020 May 15]. Available from: https:// www.who.int/news-room/fact-sheets/detail/physical-activity.

5. Heart Rhythm Society. HRS COVID-19 Task Force. [Update 2020 April 15; Accessed 2020 May 17]. Available from: https:// www.hrsonline.org/COVID19-Challenges-Solutions/hrscovid-19-task-force-update-april-15-2020.

6. Shoemaker M, Cartwright K, Hanson K, Serba D, Dickinson M, Kowalk A. Concurrent Validity of Daily Activity Data from Medtronic ICD/CRT Devices and the Actigraph GT3X Triaxial Accelerometer: A Pilot Study. Cardiopulmonary Physical Therapy Journal 2017; 28: 3-11.

7. Wilkoff BL, Auricchio A, Brugada J, Cowie M, Ellenbogen KA, Gillis AM, et al. HRS/EHRA Expert Consensus on the Monitoring of Cardiovascular Implantable Electronic Devices (CIEDs): description of techniques, indications, personnel, frequency and ethical considerations: developed in partnership with the Heart Rhythm Society (HRS) and the European Heart Rhythm Association (EHRA); and in collaboration with the American College of Cardiology (ACC), the American Heart Association (AHA), the European Society of Cardiology (ESC), the Heart Failure Association of ESC (HFA), and the Heart Failure Society of America (HFSA). Endorsed by the Heart Rhythm Society, the European Heart Rhythm Association (a registered branch of the ESC), the American College of Cardiology, the American Heart Association. Europace 2008; 10: 707-725.

8. Varma N, Epstein AE, Irimpen A, Schweikert R, Love C. Efficacy and safety of automatic remote monitoring for implantable cardioverter-defibrillator follow-up: the Lumos-T Safely Reduces Routine Office Device Follow-up (TRUST) trial. Circulation 2010; 122: 325-332.

9. Crossley GH, Boyle A, Vitense H, Chang Y, Mead RH. The CONNECT (Clinical Evaluation of Remote Notification to Reduce Time to Clinical Decision) trial: the value of wireless remote monitoring with automatic clinician alerts. J Am Coll Cardiol 2011; 57: 1181-1189.

10. Schoenfeld MH, Compton SJ, Mead RH, Weiss DN, Sherfesee L, Englund J, et al. Remote monitoring of implantable cardioverter defibrillators: a prospective analysis. Pacing Clin Electrophysiol 2004; 27 (6 Pt 1): 757-763.

11. Theuns DA, Rivero-Ayerza M, Knops P, Res JC, Jordaens L. Analysis of 57,148 transmissions by remote monitoring of implantable cardioverter defibrillators. Pacing Clin Electrophysiol 2009; 32 Suppl 1: S63-S65.

12. Lazarus A. Remote, wireless, ambulatory monitoring of implantable pacemakers, cardioverter defibrillators, and cardiac resynchronization therapy systems: analysis of a worldwide database. Pacing Clin Electrophysiol 2007; 30 (Suppl 1): S2-S12

13. Pressler A, Danner M, Esefeld K, Haller B, Scherr J, Schömig A, et al. Validity of cardiac implantable electronic devices in assessing daily physical activity. Int J Cardiol 2013; 168: 1127-1130.

14. Lancet T. COVID-19: too little, too late? The Lancet. 2020; 395: (10226).

15. Lippi G, Sanchis-Gomar F. An Estimation of the Worldwide Epidemiologic Burden of Physical Inactivity-Related Ischemic Heart Disease. Cardiovasc Drugs Ther 2020; 34: 133-137. 
16. Dickinson MG, Dam AV, Schuman E, McLeod J, Cartwright K, Schuurman C, et al. Quantification of seasonal variation in daily physical activity in individuals with heart failure and implantable cardioverter defibrillator/cardiac resynchronisation therapy devices. Heart International 2019; 13: (2).

17. Yezli S, Khan A. COVID-19 social distancing in the Kingdom of Saudi Arabia: Bold measures in the face of political, economic, social and religious challenges. Travel Medicine and Infectious Disease 2020: 21: 101692.

18. Hemphill NM, Kuan MT, Harris KC. Reduced physical activity during COVID-19 pandemic in children with congenital heart disease. Can J Cardiol 2020; 36: 1130-1134.

19. Small RS, Wickemeyer W, Germany R, Hoppe B, Andrulli $\mathrm{J}$, Brady PA, et al. Changes in intrathoracic impedance are associated with subsequent risk of hospitalizations for acute decompensated heart failure: clinical utility of implanted device monitoring without a patient alert. J Card Fail 2009; 15: 475-481.
20. Chelu MG, Gunderson BD, Koehler J, Ziegler PD, Sears SF. Patient activity decreases and mortality increases after the onset of persistent atrial fibrillation in patients with implantable cardioverter-defibrillators. JACC: Clinical Electrophysiology 2016; 2: 518-523.

21. Palmisano P, Guerra F, Ammendola E, Ziacchi M, Luigi Pisano EC, Dell'Era G, et al. Physical activity measured by implanted devices predicts atrial arrhythmias and patient outcome: Results of IMPLANTED (Italian Multicentre Observational Registry on Patients With Implantable Devices Remotely Monitored). J Am Heart Assoc 2018; 7(5): e008146.

22. Jamé S, Kutyifa V, Polonsky B, McNitt S, Al-Ahmad A, Moss AJ, et al. Predictive value of device-derived activity level for short-term outcomes in MADIT-CRT. Heart Rhythm 2017; 14: 1081-1086.

23. Kramer DB, Mitchell SL, Monteiro J, Jones PW, Normand SL, Hayes DL, et al. Patient activity and survival following Implantable Cardioverter-Defibrillator Implantation: The ALTITUDE Activity Study. J Am Heart Assoc 2015; 4 (5): $\mathrm{e} 001775$.

\section{Illustrations, Figures, Photographs}

All figures or photographs should be submitted in a high resolution (minimum 300 DPI) electronic version saved in jpeg or tiff format. Original hard copies of all figures may be requested when necessary. Photographs will be accepted at the discretion of the Editorial Board. All lettering, arrows, or other artwork must be done by an artist or draftsman. If arrows are used please ensure they appear in a different color to the background color, preferably black with a white border, or white with a black border. If arrows distinguish different items on the figure then different arrow styles should be used ie. long, short, wide, narrow. Written informed consent for publication must accompany any photograph in which the subject can be identified. Written copyright permission, from the publishers, must accompany any illustration that has been previously published. 